factors such as network/partnership risk factors can be analyzed using an egocentric survey design. These results will have future implications for targeted prevention programming in the U.S. Military.

Disclosure No significant relationships.

\section{P251 DEVELOPING PARTNER NOTIFICATION OUTCOMES FOR BACTERIAL STI BY SEX-PARTNER TYPE: INTERNATIONAL PERSPECTIVES}

${ }^{1}$ Sonali Wayal, ${ }^{1}$ Tamsin Mckinnon, ${ }^{2}$ Claudia Estcourtt, ${ }^{3}$ Nicola Low, ${ }^{1}$ Catherine Mercer, ${ }^{4}$ Merle Symonds, ${ }^{5}$ Paul Flowers, ${ }^{6}$ Jackie Cassell. ${ }^{1}$ University College London, Institute For Global Health, London, UK; ${ }^{2}$ Glasgow Caledonian University, School of Health and Life Sciences, Glasgow, UK; ${ }^{3}$ University of Bern, Institute of Social and Preventive Medicine (ISPM), Bern, Switzerland; ${ }^{4}$ Barts Health NHS Trust, London, UK; ${ }^{5}$ University of Glasgow, MRC/CSO Social and Public Health Sciences Unit, Glasgow, UK; ${ }^{6}$ Brighton and Sussex Medical School, Primary Care and Public Health, Brighton and Hove, UK

\subsection{6/sextrans-2019-sti.384}

Background Sex-partner type influences sexually transmitted infection (STI) risk. Evaluating partner notification (PN) outcomes by sex-partner type could facilitate effective targeting of resources for PN for STIs. To inform development of PN outcomes for bacterial STIs, we reviewed PN guidelines and randomised control trials (RCTs) for sex-partner type characterisation and its impact on PN outcomes.

Methods We searched online/via experts for PN guidelines worldwide and systematically reviewed RCTs of PN for bacterial STIs in PubMed to December 2018. We extracted data on $\mathrm{PN}$ recommendations and outcomes by sex-partner type.

Results We found PN guidelines from United Kingdom (UK), United States of America (USA), Canada, Australasia, Australia, and New Zealand (NZ). They recommend collecting sex-partner data using terms such as: 'regular'/'main'/'primary'/'casual'/'past'/'anonymous', without providing definitions. Australasian, NZ, Australian, and USA guidelines recommend prioritising $\mathrm{PN}$ based on factors that can enhance STI risk (e.g. having multiple partners), and emphasise PN of 'regular' partners to prevent index case re-infection. Only Australian guidelines outline auditable PN outcomes accounting for sex-partner type: index-reported number of treated 'current regular partners' or 'all past partners (includes current casual partners)'. Ten of 28 RCTs reported study participants' baseline data on sex-partner type (e.g. 'steady'/ 'regular'/'main'/'long-term'/'casual'/'one-time'), without defining them. Three RCTs reported PN outcomes by sex-partner type. Two RCTs reported higher chlamydia/gonorrhoea/trichomonas treatment rates for 'main' than 'casual' partners using expedited-partner-therapy (EPT) vs. patient-referral. Another RCT reported no difference in chlamydia re-infection rates in EPT vs. self-referral among women with a single 'steady' partner than women in overall trial.

Conclusion Current PN guidelines do not define sex-partner type nor address public health benefits of notifying different sex-partners. Sex-partner type definitions should be developed and integrated in clinical practice. RCTs should examine the effect of sex-partner types on PN outcomes. PN guidelines should account for sex-partner type based on evidence from RCTs.

Disclosure No significant relationships.

\section{P252 \\ PREDICTABILITY OF PREVALENCE OF SEXUALLY TRANSMITTED INFECTION ON COMPLEX SEXUAL NETWORK}

${ }^{1}$ Ryosuke Omori ${ }^{\star},{ }^{2}$ Laith Abu-Raddad. ${ }^{1}$ Hokkaido University, Research Center For Zoonosis Control, Sapporo, Japan; ${ }^{2}$ Weill Cornell Medicine-Qatar, Doha, Qatar

\subsection{6/sextrans-2019-sti.385}

Background Estimation of epidemic potential of a sexually transmitted infection (STI) is difficult due to difficulty in measuring and quantifying the sexual network and implications for infection transmission. We demonstrate an approach for predicting the epidemic potential of an STI using data on another STI for men who have sex with men.

Methods An individual-based mathematical model was constructed to describe sex partnering and STI concurrent transmission, namely HIV, herpes simplex virus type 2 (HSV-2), gonorrhea, chlamydia, and syphilis. The model was parameterized with representative biological and behavioral data. 500 heterogeneous sexual networks were simulated, on each of which STI transmission was also simulated. Correlations were assessed on model simulations (STI prevalences). Regressions were conducted to evaluate the predictability of HIV prevalence from each of the other STI prevalences.

Results Across these simulations, Spearman's rank correlation coefficient was 0.46 (95\% CI: $0.37-0.55)$ between HIV and HSV-2, 0.90 (95\% CI: 0.88-0.91) between HIV and gonorrhea, 0.82 (95\% CI: 0.78-0.86) between HIV and chlamydia, 0.82 (95\% CI: 0.78-0.84) between HIV and syphilis, 0.31 (95\% CI: $0.21-0.40$ ) between HSV-2 and gonorrhea, 0.82 (95\% CI: $0.78-0.86$ ) between HSV-2 and chlamydia, 0.15 (95\% CI: $0.05-0.25)$ between HSV-2 and syphilis, $0.70(95 \%$ CI: $0.65-0.75)$ between gonorrhea and chlamydia, $0.93 \quad(95 \%$ CI: 0.92-0.95) between gonorrhea and syphilis, and 0.56 (95\% CI: 0.49-0.61) between chlamydia and syphilis. The adjusted R-squared for predicting HIV prevalence using each individual STI prevalence was 0.40 for HSV-2, 0.77 for gonorrhea, 0.71 for chlamydia, and 0.57 for syphilis. The adjusted R-squared for predicting HIV prevalence in a model that includes all other STI prevalences was 0.92 .

Conclusion STI prevalence is a proxy biomarker of HIV prevalence across heterogeneous sexual networks, explaining a considerable fraction of HIV prevalence variation. However, the strength of the association between each pair of STIs varies across STIs.

Disclosure No significant relationships.

\section{P253 HPV VACCINE KNOWLEDGE AND ACCEPTABILITY AMONG MSM IN LEBANON: A QUALITATIVE STUDY}

${ }^{1}$ Ismael Maatouk*, ${ }^{2}$ Rusi Jaspal. ${ }^{1}$ Clemenceau Medical Center, Dermatology-STIS, Beirut, Lebanon; ${ }^{2}$ De Montfort University, Faculty of Life and Health Sciences, Leicester, UK

\subsection{6/sextrans-2019-sti.386}

Background Some countries specifically recommend HPV vaccination for MSM up to 26 years of age. In Lebanon, free HPV vaccine is not administered. The purpose of this exploratory study was to characterize the knowledge and beliefs of Lebanese MSM regarding HPV vaccination.

Methods Participantsthrough contacts of MSM consulting an STD clinic in Beirut-Lebanon were recruited. In-depth 